\title{
Is it possible for knowledge-based planning to improve intensity modulated radiation therapy plan quality for planners with different planning experiences in left-sided breast cancer patients?
}

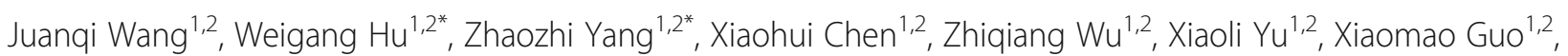
Saiquan $\mathrm{Lu}^{1,2}$, Kaixuan $\mathrm{Li}^{1,2}$ and Gongyi $\mathrm{Yu}^{1,2}$

\begin{abstract}
Background: Knowledge-based planning (KBP) is a promising technique that can improve plan quality and increase planning efficiency. However, no attempts have been made to extend the domain of KBP for planners with different planning experiences so far. The purpose of this study was to quantify the potential gains for planners with different planning experiences after implementing KBP in intensity modulated radiation therapy (IMRT) plans for left-sided breast cancer patients.
\end{abstract}

Methods: The model libraries were populated with 80 expert clinical plans from treated patients who previously received left-sided breast-conserving surgery and IMRT with simultaneously integrated boost. The libraries were created on the RapidPlan ${ }^{\mathrm{TM}}$. 6 planners with different planning experiences ( 2 beginner planners, 2 junior planners and 2 senior planners) generated manual and KBP optimized plans for additional 10 patients, similar to those included in the model libraries. The plan qualities were compared between manual and KBP plans.

Results: All plans were capable of achieving the prescription requirement. There were almost no statistically significant differences in terms of the planning target volume (PTV) coverage and dose conformality. It was demonstrated that the doses for most of organs-at-risk (OARs) were on average lower or equal in KBP plans compared to manual plans except for the senior planners, where the very small differences were not statistically significant. KBP data showed a systematic trend to have superior dose sparing at most parameters for the heart and ipsilateral lung. The observed decrease in the doses to these OARs could be achieved, particularly for the beginner and junior planners. Many differences were statistically significant.

Conclusions: It is feasible to generate acceptable IMRT plans after implementing KBP for left-sided breast cancer. KBP helps to effectively improve the quality of IMRT plans against the benchmark of manual plans for less experienced planners without any manual intervention. KBP showed promise for homogenizing the plan quality by transferring planning expertise from more experienced to less experienced planners.

Keywords: Intensity modulated radiation therapy, Knowledge-based planning, Breast cancer, Simultaneously integrated boost

\footnotetext{
*Correspondence: jackhuwg@gmail.com; yzzhi2014@163.com

'Department of Radiation Oncology, Fudan University Shanghai Cancer

Center, Shanghai, China

Full list of author information is available at the end of the article
} 


\section{Background}

Intensity modulated radiation therapy (IMRT) has the capability of maintaining the adequate planning target volume (PTV) coverage, without compromising the surrounding critical organs-at-risk (OARs) sparing. In principle, the greatest difficulty in IMRT planning is the determination of the ideal dose-volume constraints for OARs. The most common dose-volume constraints have typically been determined using the Radiation Therapy Oncology Group protocol recommendations, or a physician's intuition based recommendations. These population-based recommendations can be meaningful in many situations. However, the complex geometric variations between PTV and OARs lead to a large patient-to-patient variation in the OARs sparing. A final treatment plan that meets the guidelines and achieves the clinical acceptance criteria may still be suboptimal for a specific patient, particularly when inexperienced planners are involved. The potential of IMRT can not be fully explored until ideal IMRT optimization algorithms are widely used.

Knowledge-based planning (KBP) is a promising technique that has been demonstrated to improve plan quality and increase planning efficiency [1-12]. This technique is not intended to generate an optimal plan, which depends on other physical and clinical constrains. Rather, it aims to achieve the best plan according to the knowledge of prior plans. RapidPlan ${ }^{\mathrm{rm}}$ (Varian Medical Systems, Palo Alto, CA, USA) is a commercially KBP solution that can provide the feasibility of assessing the adequacy of OARs based on the data of clinical treated patients. The RapidPlan ${ }^{\mathrm{ma}}$ model libraries quantitatively correlate the information about anatomical geometries and dosimetry. Although the benefits of RapidPlan ${ }^{\text {Tx }}$ are still being investigated, desirable results have been reported for a range of disease sites and with varying levels of patient complexity using advanced technologies such as IMRT and volumetric modulated arc therapy (VMAT) [13-21]. In these studies, the primary focus was the appraisal of the quality, consistency and efficiency with the help of KBP. However, no attempts have been made to explore the domain of KBP for planners with different planning experiences. The IMRT planning process is demanding [22], and expertise is required to master the various steps in the procedure such as adjusting constraints and priorities to PTVs and OARs. Still, appreciable variation exists in the optimization process, depending on the experience of individual planners. For example, the commonly used simultaneous integrated boost (SIB) treatment scheme for left-sided breast cancer IMRT is quite challenging due to the complexity of the tumor bed shape, dose levels and location. These plans are especially difficult for inexperienced planners, but relatively easy for experienced planners. There is a doubt whether the effect of implementing KBP is consistent for planners with different planning experiences in varying extent. In this study, we mainly aimed to quantify the potential gains for planners with different planning experiences after implementing KBP in IMRT plans for left-sided breast cancer patients.

\section{Methods}

RapidPlan $^{\text {tw }}$, which was introduced in the Eclipse treatment planning system (TPS) from the release 13.5, is a novel knowledge-based optimization engine. It allows planners to use the dose and patient anatomy information from treated plans, in the form of dose-volume histogram (DVH) estimation model libraries, to estimate the likely amount of individual OARs sparing that can be achieved in new plans.

\section{Patient selection and contouring}

The patients who previously received left-sided breastconserving surgery and IMRT with SIB were selected for this study because breast cancer is the major cancer at our institution. The postoperative tumor bed was delineated based on the clips and seroma combined with other postoperative changes. The boost clinical target volume was defined by uniformly adding a margin of $10 \mathrm{~mm}$ around the tumor bed. An additional margin of $5 \mathrm{~mm}$ was added to define the boost PTV (PTV $\mathrm{Poost}_{\text {). }}$. The breast clinical target volume included the glandular breast tissue of the ipsilateral breast and did not extend into the pectoralis major or the ribs. The breast PTV $\left(\mathrm{PTV}_{\text {breast }}\right)$ was defined by adding a margin of $5 \mathrm{~mm}$ at the anterior-posterior direction and $10 \mathrm{~mm}$ at the medial-lateral and superior-inferior direction. Both

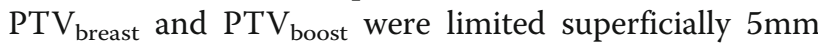
under the skin surface. For optimization and analysis

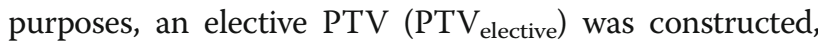
equal to $\mathrm{PTV}_{\text {breast }}$ minus $\mathrm{PTV}_{\text {boost }}$ and a transition structure of $4 \mathrm{~mm}$ between PTV $\mathrm{Poost}_{\text {b }}$ and PTV $\mathrm{V}_{\text {breast. }}$ The OARs surrounding the PTVs, including the heart, bilateral lungs, and contralateral breast were contoured as well. The heart was contoured from the level of pulmonary trunk superiorly to the apex, including the pericardium but not the major vessels.

\section{Model configuration}

The model libraries were populated with 80 expert clinical plans from treated patients between January 2012 and September 2015. These patients represented a range of breast sizes and body habitus. All plans transferred to RapidPlan $^{\mathrm{mu}}$ were originally generated with a commercial TPS (Pinnacle, version $8.0 \mathrm{~m}$, Philips Medical Systems, Fitchburg, WI) using the direct machine parameter optimization algorithm, and for the linear accelerators using 6MV photons equipped with millennium 120-leaf multileaf collimators. Each of the plans containing the geometric and dosimetric information in the model 
libraries was approved by physicians at our institution. The plans aimed to deliver $45 \mathrm{~Gy}$ to $95 \%$ of $\mathrm{PTV}_{\text {breast }}$ and $60 \mathrm{~Gy}$ to $95 \%$ of $\mathrm{PTV}_{\text {boost }}$ in 25 fractions, while limiting the volume of each PTV receiving greater than $110 \%$ of the prescribed dose. The dose constraints which were derived from our clinical experience and the published studies, are listed as follows: the percent of heart that at least received 5Gy was less than $40 \%\left(\mathrm{~V}_{5} \preccurlyeq 40 \%\right), \mathrm{V}_{20} \preccurlyeq 10 \%, \mathrm{~V}_{30} \preccurlyeq 5 \%$ and the mean dose to heart $\preccurlyeq 6 \mathrm{~Gy}$ ( $\mathrm{D}_{\text {mean }} \preccurlyeq 6 \mathrm{~Gy}$ ); ipsilateral lung $\mathrm{V}_{5} \preccurlyeq 50 \%, \mathrm{~V}_{20} \preccurlyeq 20 \%, \mathrm{~V}_{30} \preccurlyeq 5 \%$ and $\mathrm{D}_{\text {mean }} \preccurlyeq 20$ Gy; contralateral breast $D_{\text {mean }} \preccurlyeq 1 G y$ and contralateral lung $D_{\text {mean }} \preccurlyeq 1 G y$. The doses to critical OARs were not further reduced if PTV maximum dose exceeded $110 \%$ of the prescription dose, after normalizing to cover $95 \%$ of the PTV volume with the prescription dose.

\section{Evaluation of KBP performance}

The evaluation group (EG) consisted of additional 10 plans, similar to those included in the model libraries. In the comparative study, the plans manually optimized by different planners with Eclipse were compared to the plans automatically optimized with RapidPlan ${ }^{\text {Tx. }}$. RapidPlan $^{\mathrm{Tx}}$ optimized plans will be defined as KBP plans, while the plans generated with Eclipse without the help of RapidPlan ${ }^{\text {ma }}$ are defined as manual plans. Optimization and dose calculation were performed using the progressive resolution optimizer and the anisotropic analytical algorithm with a $2.5 \mathrm{~mm}$ calculation grid. The plans included in EG were not included in the model libraries. Both IMRT plans were generated with the same prescription dose and beam characteristics (beam isocenter, angles and numbers). During the study, 6 planners $(2$ beginner planners, 2 junior planners and 2 senior planners) with planning experience of 2-10 years interested in the study were engaged in the IMRT planning. One year planning experience at our institution may be equivalent to several years' experience at other institutions, because we annually treated more than one thousand breast cancer patients. One beginner planner, junior planner and senior planner have generated approximately 120, 600 and more than 1200 breast cancer IMRT plans, respectively.

\section{Dose comparison}

All plans were evaluated and compared by a physician who specializes in breast cancer RT with more than 20 years work experience for their ability to fulfill the plan acceptance criteria. DVHs were calculated for each plan. For PTV $\mathrm{Plective}_{\text {and }} \mathrm{PTV}_{\text {boost }}$, the lowest dose received by $98 \%$ of the volume $\left(D_{98 \%}\right)$ and the highest dose received by $2 \%$ of the volume $\left(D_{2 \%}\right)$ were compared. The dose conformality of PTVs was evaluated by the conformation number $(\mathrm{CN})$ according to the following equation (1):

$$
\mathrm{CN}=\frac{\mathrm{PTV}_{\text {ref }}^{2}}{\mathrm{~V}_{\mathrm{PTV}} \times \mathrm{V}_{\text {ref }}},
$$

where $\mathrm{PTV}_{\text {ref }}$ represented the volume receiving the prescription dose in PTV, $\mathrm{V}_{\mathrm{PTV}}$ stood for the volume of the PTV, and $V_{\text {ref }}$ was the volume that received the prescribed dose. For OARs, $D_{\text {mean }}$ and $V_{x}$ to the heart $(x=5,20$ and 30), ipsilateral lung $(x=5,20$ and 30), contralateral breast and contralateral lung were compared. The plan difference was facilitated by defining the relative OAR dose deduction $(\delta)$ according to the following equation (2):

$$
\delta=\frac{\mathrm{P}_{\text {manual }}-\mathrm{P}_{\mathrm{KBP}}}{\mathrm{P}_{\text {manual }}} \times 100,
$$

where P refers to the dose (Gy) or volume (\%).

\section{Statistical analysis}

Statistical analysis was performed to compare the dosimetric differences between manual and KBP plans. Kolmogorov-Smirnov tests for the normal distribution were run for all relevant metrics of all structures. The paired $\mathrm{t}$-test was run for each relevant metric for each planner. There were 36 tests run in total for PTVs and 60 tests for OARs. In consideration of test multiplicity, a $p$-value $<0.0014$ was considered statistically significant for PTVs and $<0.00083$ for OARs after the Bonferroni correction. All statistical analyses were performed with IBM-SPSS statistics, version 19 (SPSS Inc, Chicago, IL).

\section{Results \\ PTV coverage}

Table 1 presents the detailed statistical analysis of the PTV coverage and $\mathrm{CN}$ for the entire EG divided per planner. The dose distributions for one representative patient on coronal planes of manual and KBP plans are shown in Fig. 1(a) and (b) for one beginner planner, Fig. 1(e) and (f) for one junior planner and Fig. 1(i) and (j) for one senior planner, respectively. All plans were capable of achieving the prescription requirement. There were almost no statistically significant differences in terms of the PTV coverage, while the $\mathrm{CN}$ was remarkably similar. It clearly showed that the PTV dose distributions were essentially equivalent between the two plans.

\section{OARs sparing}

The summary of the quantitative analysis of the OARs sparing for the entire EG is listed in Table 2. Figure 1(d), (h) and (l) show the comparisons of DVHs for one representative patient, respectively. The distributions of all DVH metrics were sufficiently similar to normal distributions. 
Table 1 The detailed statistical analysis of the PTV coverage and CN for the entire evaluation group divided per planner

\begin{tabular}{|c|c|c|c|c|c|c|c|}
\hline \multirow[t]{2}{*}{ Planner } & \multirow[t]{2}{*}{ Plan } & \multicolumn{3}{|l|}{ PTV boost } & \multicolumn{3}{|l|}{ PTV elective } \\
\hline & & $\mathrm{D}_{98 \%}(\mathrm{~Gy})$ & $D_{2 \%}(G y)$ & $\mathrm{CN}$ & $\mathrm{D}_{98 \%}(\mathrm{~Gy})$ & $D_{2 \%}(G y)$ & $\mathrm{CN}$ \\
\hline \multirow[t]{3}{*}{ Beginner planner $\mathrm{A}$} & Manual plan & 59.54 & 63.77 & 0.70 & 45.39 & 57.69 & 0.65 \\
\hline & KBP plan & 59.45 & 63.95 & 0.72 & 45.37 & 57.86 & 0.66 \\
\hline & $p$ value & 0.06 & 0.20 & 0.07 & 0.79 & 0.39 & 0.38 \\
\hline \multirow[t]{3}{*}{ Beginner planner B } & Manual plan & 59.43 & 63.79 & 0.73 & 45.81 & 57.75 & 0.65 \\
\hline & KBP plan & 59.38 & 64.16 & 0.73 & 45.66 & 57.35 & 0.70 \\
\hline & $p$ value & 0.52 & 0.13 & 0.38 & 0.40 & 0.33 & 0.05 \\
\hline \multirow[t]{3}{*}{ Junior planner A } & Manual plan & 59.35 & 65.24 & 0.74 & 45.15 & 56.88 & 0.69 \\
\hline & KBP plan & 59.19 & 65.34 & 0.73 & 45.09 & 56.85 & 0.68 \\
\hline & $p$ value & 0.24 & 0.82 & 0.14 & 0.72 & 0.91 & 0.20 \\
\hline \multirow[t]{3}{*}{ Junior planner B } & Manual plan & 59.53 & 63.63 & 0.74 & 44.95 & 58 & 0.69 \\
\hline & KBP plan & 59.33 & 63.53 & 0.73 & 44.55 & 58.39 & 0.69 \\
\hline & $p$ value & 0.17 & 0.55 & 0.44 & 0.05 & 0.16 & 0.88 \\
\hline \multirow[t]{3}{*}{ Senior planner A } & Manual plan & 59.43 & 64.57 & 0.77 & 45.85 & 57.4 & 0.68 \\
\hline & KBP plan & 59.44 & 64.91 & 0.75 & 45.91 & 58.12 & 0.69 \\
\hline & $p$ value & 0.86 & 0.07 & 0.06 & 0.76 & 0.11 & 0.17 \\
\hline \multirow[t]{3}{*}{ Senior planner B } & Manual plan & 59.42 & 64.85 & 0.72 & 45.92 & 57.18 & 0.70 \\
\hline & KBP plan & 59.43 & 64.06 & 0.74 & 46.14 & 57.94 & 0.70 \\
\hline & $p$ value & 0.93 & 0.08 & 0.23 & 0.08 & 0.05 & 0.37 \\
\hline
\end{tabular}

Data shown are the averages of the respective parameters for the 10 patients
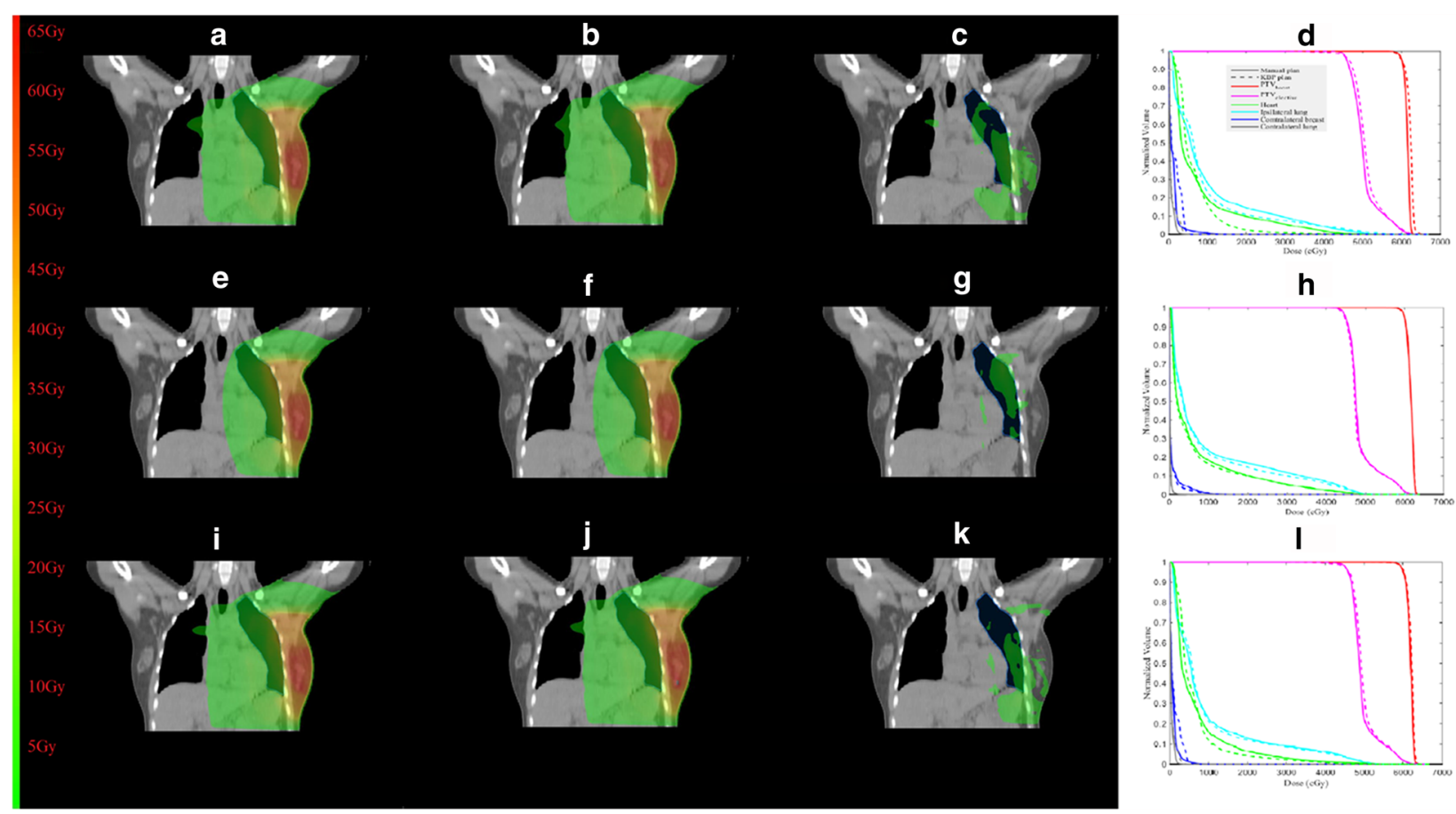

$\mathbf{h}$
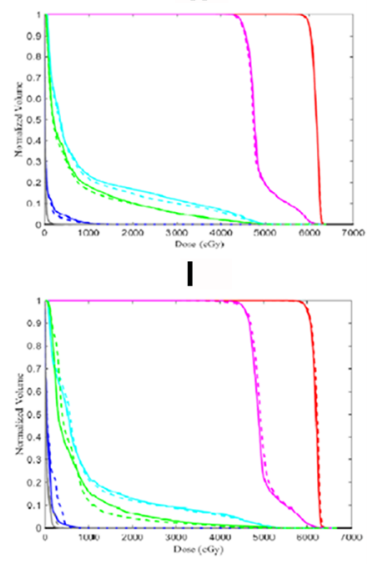

Fig. 1 The dose distributions for one representative patient on coronal planes of manual and KBP plans are shown in (a) and (b) for one beginner planner, (e) and (f) for one junior planner and (i) and (j) for one senior planner, respectively. (c), (g) and (k) display the dose differences between $(\mathbf{a})$ and $(\mathbf{b}),(\mathbf{e})$ and $(\mathbf{f})$ and $(\mathbf{i})$ and $(\mathbf{j})$, respectively. The dose-volume histograms are shown for $(\mathbf{d})$ one beginner planner, (h) one junior planner and (I) one senior planner 
Table 2 The summary of the quantitative analysis of the OARs sparing for the entire evaluation group for each planner

\begin{tabular}{|c|c|c|c|c|c|c|c|c|c|c|c|}
\hline \multirow[t]{2}{*}{ Planner } & \multirow[t]{2}{*}{ Plan } & \multicolumn{4}{|l|}{ Heart } & \multicolumn{4}{|c|}{ Ipsilateral lung } & \multirow{2}{*}{$\begin{array}{l}\text { Contralateral } \\
\text { breast } \\
\text { Mean } \\
\text { dose (Gy) }\end{array}$} & \multirow{2}{*}{$\begin{array}{l}\text { Contralatera } \\
\text { lung } \\
\text { Mean } \\
\text { dose (Gy) }\end{array}$} \\
\hline & & $\mathrm{V}_{5}(\%)$ & $V_{20}(\%)$ & $V_{30}(\%)$ & $\begin{array}{l}\text { Mean } \\
\text { dose (Gy) }\end{array}$ & $\mathrm{V}_{5}(\%)$ & $V_{20}(\%)$ & $V_{30}(\%)$ & $\begin{array}{l}\text { Mean } \\
\text { dose (Gy) }\end{array}$ & & \\
\hline \multirow[t]{3}{*}{ Beginner planner A } & Manual plan & 39.80 & 10.89 & 5.73 & 7.50 & 58.13 & 18.42 & 12.09 & 11.60 & 0.43 & 0.20 \\
\hline & KBP plan & 36.87 & 7.49 & 4.23 & 6.62 & 51.56 & 16.59 & 11.15 & 11.43 & 0.47 & 0.20 \\
\hline & $p$ value & 0.002 & $<0.0001$ & 0.001 & $<0.0001$ & $<0.0001$ & 0.001 & 0.01 & 0.33 & 0.32 & 0.95 \\
\hline \multirow[t]{3}{*}{ Beginner planner B } & Manual plan & 39.37 & 11.28 & 6.03 & 7.52 & 58.15 & 18.31 & 12.12 & 11.85 & 0.47 & 0.20 \\
\hline & KBP plan & 37.73 & 7.80 & 4.13 & 6.63 & 53.46 & 16.21 & 10.52 & 11.47 & 0.48 & 0.16 \\
\hline & $p$ value & 0.30 & $<0.0001$ & $<0.0001$ & $<0.0001$ & 0.01 & 0.002 & 0.004 & 0.21 & 0.20 & 0.06 \\
\hline \multirow[t]{3}{*}{ Junior planner A } & Manual plan & 36.46 & 10.25 & 5.33 & 6.99 & 52.3 & 19.16 & 12.82 & 11.70 & 0.49 & 0.17 \\
\hline & KBP plan & 33.85 & 9.73 & 5.30 & 6.61 & 50.52 & 18.88 & 12.49 & 11.51 & 0.43 & 0.17 \\
\hline & $p$ value & $<0.0001$ & 0.04 & 0.87 & $<0.0001$ & 0.03 & 0.32 & 0.44 & 0.41 & 0.18 & 0.17 \\
\hline \multirow[t]{3}{*}{ Junior planner B } & Manual plan & 35.36 & 10.41 & 5.54 & 7.00 & 53.75 & 18.56 & 11.94 & 11.78 & 0.41 & 0.17 \\
\hline & KBP plan & 34.63 & 9.63 & 5.20 & 6.6 & 51.92 & 17.98 & 11.75 & 11.41 & 0.41 & 0.17 \\
\hline & $p$ value & 0.23 & 0.02 & 0.04 & $<0.0001$ & 0.02 & 0.10 & 0.42 & 0.05 & 0.88 & 0.82 \\
\hline \multirow[t]{3}{*}{ Senior planner A } & Manual plan & 36.58 & 8.00 & 4.53 & 6.72 & 53.76 & 18.51 & 12.76 & 11.79 & 0.46 & 0.22 \\
\hline & KBP plan & 37.80 & 7.76 & 4.64 & 6.63 & 54.42 & 18.63 & 12.73 & 11.94 & 0.47 & 0.19 \\
\hline & $p$ value & 0.06 & 0.23 & 0.41 & 0.14 & 0.39 & 0.70 & 0.91 & 0.48 & 0.24 & 0.27 \\
\hline \multirow[t]{3}{*}{ Senior planner B } & Manual plan & 36.78 & 7.98 & 4.59 & 6.70 & 54.07 & 17.97 & 12.16 & 11.74 & 0.47 & 0.21 \\
\hline & KBP plan & 37.00 & 7.74 & 4.58 & 6.55 & 54.32 & 17.8 & 12.07 & 11.73 & 0.47 & 0.19 \\
\hline & $p$ value & 0.71 & 0.40 & 0.90 & 0.02 & 0.77 & 0.60 & 0.68 & 0.98 & 0.70 & 0.15 \\
\hline
\end{tabular}

Data shown are the averages of the respective parameters for the 10 patients

Overall, the results demonstrated that the doses to most of OARs were on average lower or equal in KBP plans compared to manual plans except for the senior planners, where the very small differences were not statistically significant. Notably, KBP data showed a systematic trend to have superior dose sparing at most parameters for the heart and ipsilateral lung without adding doses to the other OARs or compromising the PTV coverage. The improved OARs sparing could be achieved, particularly for the beginner and junior planners. Many differences were statistically significant. Besides, the variability in the doses to all OARs was effectively reduced between KPB plans generated by planners with different planning experiences.

Figure $1(\mathrm{c}),(\mathrm{g})$ and $(\mathrm{k})$ display the dose differences between Fig. 1(a), (b), (e), (f), (i) and (j), respectively. The major dose differences are mainly caused by adoption of KBP which led to varying degrees of improved OARs sparing for different planners. Figure 2 shows the effect of KBP implementation on (a) the heart and (b) the ipsilateral lung dose for planners with each level of planning experiences. On average, the heart $\delta$ for the beginner planners was $5.1 \%$ for $\mathrm{V}_{5}, 32.3 \%$ for $\mathrm{V}_{20}, 29.4 \%$ for $\mathrm{V}_{30}$ and $10.9 \%$ for $D_{\text {mean }}$, respectively; while for the junior planners was $4.7 \%$ for $V_{5}, 6.46 \%$ for $V_{20}, 2.9 \%$ for $V_{30}$ and $5.1 \%$ for $\mathrm{D}_{\text {mean }}$, respectively. For the ipsilateral lung, the average $\delta$ for the beginner planners was $9.5 \%$ for $V_{5}$,
$10.7 \%$ for $V_{20}, 10.4 \%$ for $V_{30}$ and $2.0 \%$ for $D_{\text {mean }}$, respectively; while for the junior planners was $2.9 \%$ for $\mathrm{V}_{5}, 2.3 \%$ for $\mathrm{V}_{20}, 1.9 \%$ for $\mathrm{V}_{30}$ and $2 \%$ for $\mathrm{D}_{\text {mean }}$, respectively. For the senior planners, KBP was found to have no noticeable effect on these OARs sparing.

\section{Discussion}

This study demonstrated the feasibility of implementing KBP to assist IMRT planning for left-sided breast cancer patients. This is in line with Fogliata et al., pioneers in implementing KBP in breast cancer, who provided the evidence about the dosimetric quality and effectiveness [19]. Furthermore, we quantified the potential gains of KBP for planners with different planning experiences. KBP proved to be useful in guiding the beginner and junior planners to improve the plan quality without any manual intervention. Based on our results, KBP plans provided at least comparable plan quality compared to manual ones. In addition, adoption of KBP was able to improve the consistency of IMRT plans by minimizing the variations of plan quality due to individual planner differences. KBP showed promise for homogenizing plan quality by transferring planning expertise from more experienced to less experienced planners. Moreover, our results also showed that the experienced planners derived fewer benefits from KBP. 

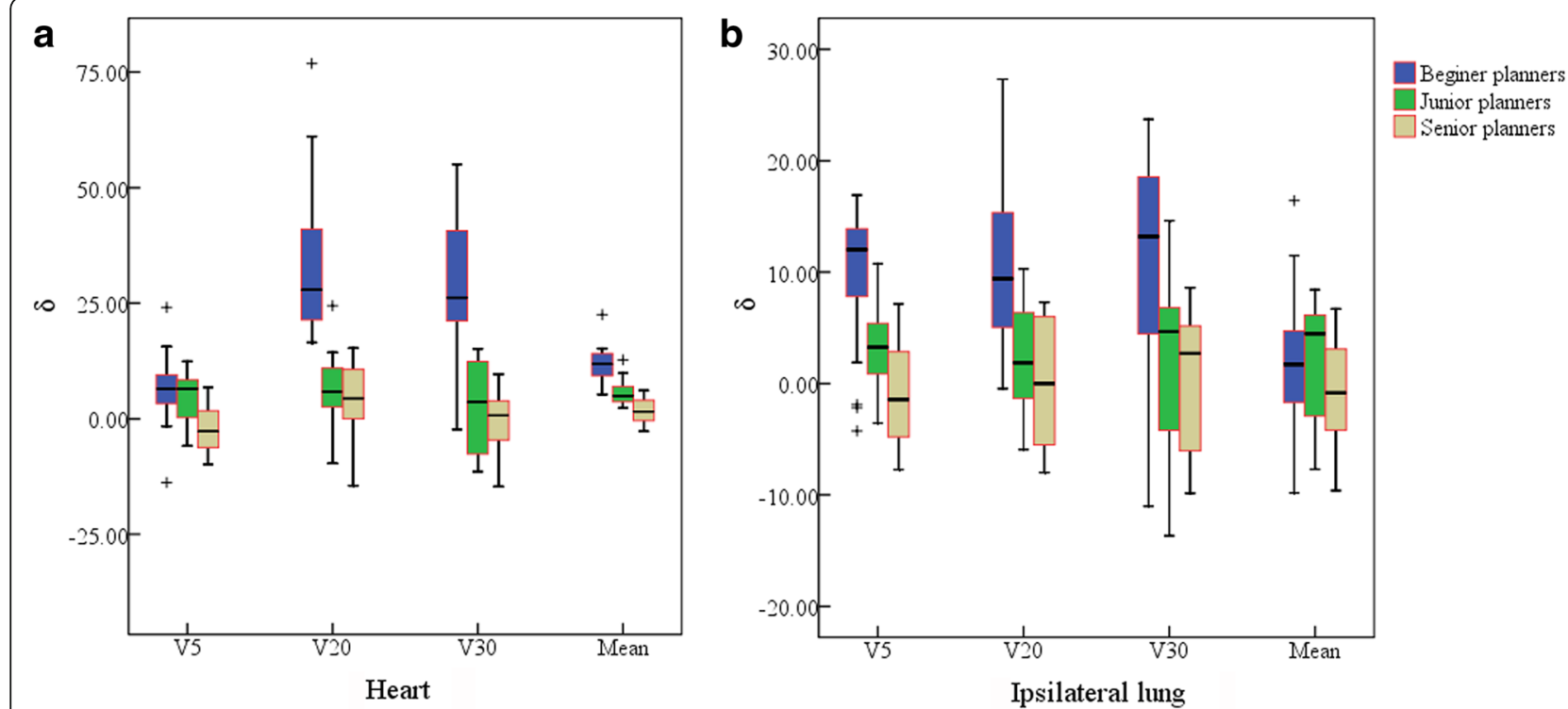

Fig. 2 The box plots depicting the effect of KBP implementation on (a) the heart and (b) the ipsilateral lung dose for planners with each level of planning experiences. $\delta$ means the relative OAR dose deduction

Left-sided breast cancer patients received IMRT with SIB were chosen for the analysis. Besides the prevalence of the cancer, there are multiple PTVs and many individual OARs that need to be balanced. Therefore, we considered it was an appropriate clinical scenario to test the performance of KBP, for relatively demanding plans. The development and implementation of heart-sparing breast RT techniques remains an international priority. Breathhold techniques [23-28] and VMAT [28] improve the heart sparing. Both techniques might be advisable for breast RT, but they have not yet been widely implemented at our institution due to resource costs and staff training. The study was limited to IMRT delivered to free-breathing patients.

Our data showed that KBP plans were similar to manual ones in the PTV coverage and dose conformality. Most importantly, the doses to most of OARs were on average lower or equal in KBP plans compared to manual ones. The finding of improved OARs sparing could be explained by suboptimal dose-volume constraints being used in the historical manual-planning cohort. Encompassing the breast plus tumor bed without increasing the risks of cardiac complication probabilities is a great technical challenge when the surgical bed location is close to the heart. Fifteen-year cardiac mortality was higher for patients who received RT for left-sided compared to right-sided breast cancer [29]. Patients with early-stage breast cancer have a high probability of long-term survival; parts of the survival gain could be offset by the non-breast related mortality and morbidity from late cardiovascular damage due to the cardiac exposure to radiation in the long time follow-up. Therefore, the critical goal of breast RT should always be to minimize the irradiated heart volume as much as possible (without compromising PTV coverage). Decreases of heart $\mathrm{D}_{\text {mean }}$ by more than $10 \%$ with KBP were found in $25 \%$ of the plans. Darby et al. [30] showed a linear increase in the relative rate of major coronary events with the heart $D_{\text {mean }}$. $7.4 \% / G y$ is the excess relative risk per Gy. Based on this, a reduction of $0.89 \mathrm{~Gy}$ for the beginner planners could represent an approximately $6.6 \%$ improvement in longterm major coronary events and a reduction of $0.39 \mathrm{~Gy}$ for the junior planners could represent an approximately $3.0 \%$ improvement. It should be stressed, however, that this is highly patient-specific and will be influenced by other factors. More than $45 \%$ patients had the relative reduction of heart $\mathrm{V}_{30}$ by more than $10 \%$ with KBP. Previous studies [31, 32] of early breast cancer and Hodgkin's disease have shown that the heart $\mathrm{V}_{30}$ may also result in few cardiac complications. However, looking over the studies [30,33] of radiation-related heart disease of breast cancer patients and atomic bomb survivors, there are no known "safe" levels of radiation to the heart. Therefore, the heart should be spared as much as possible. Furthermore, the ipsilateral lung sparing is an additional concern for breast cancer patients. The quantitative analysis of normal tissue effects in the clinic project reviewed several dose-response studies for lung, and found that there were usually strong correlations between various dosimetric parameters (e.g., $\mathrm{V}_{5}$ and $\mathrm{V}_{20}$ ) and pneumonitis risk [34]. Our results suggested that KBP plans were superior to manual ones in ipsilateral lung $V_{20}$ especially for the beginner planners. 
Various alternative solutions are being proposed to assist planners efficiently guiding clinical tradeoffs among the competing objectives (PTV coverage, OARs sparing and practical feasibility) and decide the clinically optimal balance, such as AutoPlanning [35] and multicriteria optimization [36-42]. AutoPlanning aims to use an iterative algorithm based approach to automatically adapt objectives, constraints and dose shaping structures during the planning optimization process to achieve clinical goals. Multicriteria optimization starts from static clinical objectives and navigates through the realm of possible solutions to find the Pareto optimal plan. Both approaches are timeconsuming and also require profound knowledge, experience and interaction to determine the optimal plan, which poses special difficulty of correlating the anatomy and dosimetry relations.

One limitation of the current study is that IMRT plans in the model libraries were generated with Pinnacle ${ }^{3}$ and the new ones with Eclipse. The large difference between the optimization algorithm and dose calculation may affect the estimations and statements about the accuracy of the model libraries. Another limitation is the range of patient geometries in the model libraries, which still may not represent the full diversity of breast cancer cases due to individual differences. Special caution should be taken when applying the model libraries to those patients whose geometry falls outside the range of the constituent plans in the libraries. In addition, the estimated DVH curves imply the information about physicianapproved dose-volume constraints and represent acceptable tradeoffs for the specific patient. The accuracy of the estimations mainly depends on the quality of IMRT plans in the model libraries. However, the optimization may be compromised to a certain extent due to the planning complexity and other clinical constraints. Suboptimal plans in the model libraries may degrade results with the KBP approach. Deeper analyses on the goodness of the estimation model configuration in terms of the model size, plan and anatomy homogeneity are required in the future.

\section{Conclusions}

It is feasible to generate acceptable IMRT plans after implementing KBP for left-sided breast cancer. KBP helps to effectively improve the quality of IMRT plans against the benchmark of manual plans for less experienced planners without any manual intervention. KBP showed promise for homogenizing the plan quality by transferring planning expertise from more experienced to less experienced planners. More training and fine-tuning might further improve the performance of KBP, which will be further explored at our institution.

\section{Abbreviations}

$\mathrm{CN}$ : The conformation number; $\mathrm{D}_{2} \%$ : The highest dose received by $2 \%$ of the volume; $D_{98 \%}$ : The lowest dose received by $98 \%$ of the volume; $D_{\text {mean }}$ : The mean dose; DVH: Dose-volume histogram; EG: Evaluation group;

IMRT: Intensity modulated radiotherapy; KBP: Knowledge-based planning; OAR: Organ-at-risk; P: The dose or volume of organ-at-risk; PTV: Planning target volume; PTV boost: The boost PTV; PTV breast: The breast PTV;

PTV elective: The elective PTV; PTV ref: The volume receiving the prescription dose in the target volume; SIB: Simultaneous integrated boost;

TPS: Treatment planning system; VMAT: Volumetric modulated arc therapy; $V_{\text {PTV: }}$ The volume of the PTV; $V_{\text {ref. }}$ The volume that received the prescribed dose; $V_{x}$ : The percent of volume that at least received $x G y ; \delta$ : The relative dose deduction

\section{Acknowledgements}

Not applicable.

\section{Funding}

This work was supported by the National Natural Science Foundation of China (Grant No. 81372430 and 81402525) and the Municipal Natural Science Foundation of Shanghai (Grant No. 16ZR1406900).

Availability of data and materials

The original data will not be shared according to the department rules.

\section{Authors' contributions}

JW created the DVH estimation model analyzed all plans that were used in this study and drafted the manuscript. JW, XC, ZW, SL, KL and GY planned for treatment cases. $X Y$ and $X G$ contributed to the data collection. $\mathrm{WH}$ and $Z Y$ designed the study and composed the manuscript. All authors read and approved the final manuscript.

Competing interests

The authors declare that they have no competing interests.

Consent for publication

Not applicable.

Ethics approval and consent to participate

Not applicable.

\section{Publisher's Note}

Springer Nature remains neutral with regard to jurisdictional claims in published maps and institutional affiliations.

\section{Author details}

${ }^{1}$ Department of Radiation Oncology, Fudan University Shanghai Cancer Center, Shanghai, China. ${ }^{2}$ Department of Oncology, Shanghai Medical College, Fudan University, Shanghai, China.

Received: 13 September 2016 Accepted: 15 May 2017

Published online: 22 May 2017

References

1. Wu B, Ricchetti F, Sanguineti G, Kazhdan M, Simari P, Chuang M, et al. Patient geometry-driven information retrieval for IMRT treatment plan quality control. Med Phys. 2009;36:5497-505.

2. Wu B, Ricchetti F, Sanguineti G, Kazhdan M, Simari P, Jacques R, et al. Datadriven approach to generating achievable dose-volume histogram objectives in intensity-modulated radiotherapy planning. Int J Radiat Oncol Biol Phys. 2011;79:1241-7.

3. Lian J, Yuan L, Ge Y, Chera BS, Yoo DP, Chang S, et al. Modeling the dosimetry of organ-at-risk in head and neck IMRT planning: an intertechnique and interinstitutional study. Med Phys. 2013;40:121704.

4. Nelms BE, Robinson G, Markham J, Velasco K, Boyd S, Narayan S, et al. Variation in external beam treatment plan quality: An inter-institutional study of planners and planning systems. Pract Radiat Oncol. 2012;2:296-305.

5. Moore KL, Brame RS, Low DA, Mutic S. Experience based quality control of clinical intensity modulated radiotherapy planning. Int J Radiat Oncol Biol Phys. 2011;81:545-51. 
6. Zhu X, Ge Y, Li T, Thongphiew D, Yin FF, Wu QJ. A planning quality evaluation tool for prostate adaptive IMRT based on machine learning. Med Phys. 2011;38:719-26.

7. Chanyavanich V, Das SK, Lee WR, Lo JY. Knowledge based IMRT treatment planning for prostate cancer. Med Phys. 2011;38:2515-22.

8. Yuan L, Ge Y, Lee WR, Yin FF, Kirkpatrick JP, Wu QJ. Quantitative analysis of the factors which affect the inter-patient organ-at risk dose sparing variation in IMRT plans. Med Phys. 2012;39:6868-78.

9. Petit SF, Wu B, Kazhdan M, Dekker A, Simari P, Kumar R, et al. Increased organ sparing using shape-based treatment plan optimization for intensity modulated radiation therapy of pancreatic adenocarcinoma. Radiother Oncol. 2012;102:38-44.

10. Appenzoller LM, Michalski JM, Thorstad WL, Mutic S, Moore KL. Predicting dosevolume histograms for organs-at-risk in IMRT planning. Med Phys. 2012:39:7446-61

11. Good D, Lo J, Lee WR, Wu QJ, Yin FF, Das SK. A knowledge-based approach to improving and homogenizing intensity modulated radiation therapy planning quality among treatment centers: an example application to prostate cancer planning. Int J Radiat Oncol Biol Phys. 2013;87:176-81.

12. Schmidt M, Lo JY, Grzetic S, Lutzky C, Brizel DM, Das SK. Semiautomated head-and-neck IMRT planning using dose warping and scaling to robustly adapt plans in a knowledge database containing potentially suboptimal plans. Med Phys. 2015;42:4428-34.

13. Wu B, McNutt T, Zahurak M, Simari P, Pang D, et al. Fully automated simultaneous integrated boosted-intensity modulated radiation therapy treatment planning is feasible for head-and-neck cancer: a prospective clinical study. Int J Radiat Oncol Biol Phys. 2012;84:e647-53.

14. Fogliata A, Wang PM, Belosi F, Clivio A, Nicolini G, Vanetti E, et al, Assessment of a model based optimization engine for volumetric modulated arc therapy for patients with advanced hepatocellular cancer. Radiat Oncol. 2014;9:236.

15. Fogliata A, Belosi F, Clivio A, Navarria P, Nicolini G, Scorsetti M, et al. On the pre-clinical validation of a commercial model-based optimisation engine: application to volumetric modulated arc therapy for patients with lung or prostate cancer. Radiother Oncol. 2014;113:385-91.

16. Tol JP, Delaney AR, Dahele M, Slotman BJ, Verbakel WF. Evaluation of a knowledge-based planning solution for head and neck cancer. Int J Radiat Oncol Biol Phys. 2015;91:612-20.

17. Tol JP, Dahele M, Delaney AR, Slotman BJ, Verbakel WF. Can knowledgebased DVH predictions be used for automated, individualized quality assurance of radiotherapy treatment plans? Radiat Oncol. 2015;10:234

18. Fogliata A, Nicolini G, Clivio A, Vanetti E, Laksar S, Tozzi A. A broad scope knowledge based model for optimization of VMAT in esophageal cancer: validation and assessment of plan quality among different treatment centers. Radiat Oncol. 2015;10:220.

19. Fogliata A, Nicolini G, Bourgier C, Clivio A, De Rose F, Fenoglietto P, et al. Performance of a Knowledge-Based Model for Optimization of Volumetric Modulated Arc Therapy Plans for Single and Bilateral Breast Irradiation. PLoS One. 2015:10:e0145137.

20. Wu H, Jiang F, Yue H, Zhang H, Wang K, Zhang Y. Applying a RapidPlan model trained on a technique and orientation to another: a feasibility and dosimetric evaluation. Radiat Oncol. 2016;11:108.

21. Chang AT, Hung AW, Cheung FW, Lee MC, Chan OS, Philips H, et al. Comparison of Planning Quality and Efficiency Between Conventional and Knowledge-based Algorithms in Nasopharyngeal Cancer Patients Using Intensity Modulated Radiation Therapy. Int J Radiat Oncol Biol Phys. 2016;95:981-90

22. Webb S. The physical basis of IMRT and inverse planning. Br J Radiol. 2003; 76:678-89.

23. Remouchamps VM, Vicini FA, Sharpe MB, Kestin LL, Martinez AA, Wong JW. Significant reductions in heart and lung doses using deep inspiration breath hold with active breathing control and intensity-modulated radiation therapy for patients treated with locoregional breast irradiation. Int J Radiat Oncol Biol Phys. 2003;55:392-406.

24. Stranzl H, Zurl B, Langsenlehner T, Kapp KS. Wide tangential fields including the internal mammary lymph nodes in patients with left-sided breast cancer. Influence of respiratory-controlled radiotherapy (4D-CT) on cardiac exposure. Strahlenther Onkol. 2009:185:155-60.

25. Borst GR, Sonke JJ, den Hollander S, Betgen A, Remeijer P, van Giersbergen $A$, et al. Clinical results of image-guided deep inspiration breath hold breast irradiation. Int J Radiat Oncol Biol Phys. 2010;78:1345-51.
26. Hjelstuen MH, Mjaaland I, Vikström J, Dybvik Kl. Radiation during deep inspiration allows loco-regional treatment of left breast and axillary-, supraclavicular- and internal mammary lymph nodes without compromising target coverage or dose restrictions to organs at risk. Acta Oncol. 2012:51:333-44.

27. Nissen HD, Appelt AL. Improved heart, lung and target dose with deep inspiration breath hold in a large clinical series of breast cancer patients. Radiother Oncol. 2013;106:28-32.

28. Osman SO, Hol S, Poortmans PM, Essers M. Volumetric modulated arc therapy and breath-hold in image-guided locoregional left-sided breast irradiation. Radiother Oncol. 2014;112:17-22.

29. Clarke M, Collins R, Darby S, Davies C, Elphinstone P, Evans V, et al. Effects of radiotherapy and of differences in the extent of surgery for early breast cancer on local recurrence and 15-year survival: an overview of the randomised trials. Lancet. 2005;366:2087-106.

30. Darby SC, Ewertz M, McGale P, Bennet AM, Blom-Goldman U, Brønnum D, et al. Risk of ischemic heart disease in women after radiotherapy for breast cancer. N Engl J Med. 2013;368:987-98.

31. Reinders JG, Heijmen BJ, Olofsen-van Acht MJ, van Putten WL, Levendag PC Ischemic heart disease after mantlefield irradiation for Hodgkin's disease in long-term follow-up. Radiother Oncol. 1999;51:35-42.

32. Venables K, Miles EA, Deighton A, Aird EG, Hoskin PJ. Irradiation of the heart during tangential breast treatment: a study within the START trial. Br J Radiol. 2004;77:137-42

33. Preston DL, Shimizu Y, Pierce DA, Suyama A, Mabuchi K. Studies of mortality of atomic bomb survivors. Report 13: Solid cancer and noncancer disease mortality: 1950-1997. Radiat Res. 2003:160:381-407.

34. Marks LB, Bentzen SM, Deasy JO, Kong FM, Bradley JD, Vogelius IS, et al. Radiation dose-volume effects in the lung. Int J Radiat Oncol Biol Phys. 2010;76:570-6.

35. Krayenbuehl J, Norton I, Studer G, Guckenberger M. Evaluation of an automated knowledge based treatment planning system for head and neck. Radiat Oncol, 2015:10:226

36. Craft DL, Halabi TF, Shih HA, Bortfeld TR. Approximating convex pareto surfaces in multiobjective radiotherapy planning. Med Phys. 2006:33:3399-407.

37. Thieke C, Küfer KH, Monz M, Scherrer A, Alonso F, et al. A new concept for interactive radiotherapy planning with multicriteria optimization: first clinical evaluation. Radiother Oncol. 2007;85:292-8.

38. Monz M, Küfer KH, Bortfeld TR, Thieke C. Pareto navigation: algorithmic foundation of interactive multi-criteria IMRT planning. Phys Med Biol. 2008;53:985-98.

39. Hong TS, Craft DL, Carlsson F, Bortfeld TR. Multicriteria optimization in intensity-modulated radiation therapy treatment planning for locally advanced cancer of the pancreatic head. Int J Radiat Oncol Biol Phys. 2008;72:1208-14.

40. Spalke T, Craft D, Bortfeld T. Analyzing the main trade-offs in multiobjective radiation therapy treatment planning databases. Phys Med Biol. 2009;54:3741-54.

41. Hu W, Wang J, Li G, Peng J, Lu S, Zhang Z. Investigation of plan quality between RapidArc and IMRT for gastric cancer based on a novel beam angle and multicriteria optimization technique. Radiother Oncol. 2014:111:144-7.

42. Kierkels RG, Visser R, Bijl HP, et al. Multicriteria optimization enables less experienced planners to efficiently produce high quality treatment plans in head and neck cancer radiotherapy. Radiat Oncol. 2015;10:87. 